\title{
Effects of Ventilatory Settings on Pendelluft Phenomenon During Mechanical Ventilation
}

\author{
Yusuke Enokidani, Akinori Uchiyama, Takeshi Yoshida, Ryuichiro Abe, Tomonori Yamashita, \\ Yukiko Koyama, and Yuji Fujino
}

\begin{abstract}
BACKGROUND: Pendelluft phenomenon is defined as the displacement of gas from a more recruited nondependent (ND) lung region to a less recruited dependent (D) lung region. This phenomenon may cause lung injury. Thus, a lung model for pendelluft was established, and the effects of ventilatory settings on pendelluft were examined. METHODS: Two sets of the twinbellows-type training test lung (TTL) model were utilized. One set of bellows simulated the diaphragm, and the other simulated the lung. One TTL model represented the ND region, and the other represented the $\mathrm{D}$ region. The lung bellows were connected to each other and were ventilated with 1 ventilator. The diaphragm bellows were ventilated with 2 synchronized ventilators that regulated pleural pressure levels. We simulated pendelluft by applying different pleural pressure levels to the $\mathrm{D}$ and ND bellows. The increment of the tidal volume in the $\mathrm{D}$ region from the "no breathing effort" condition was defined as the pendelluft volume. The effects of ventilator settings, such as ventilatory modes, triggering sensitivity, inspiratory pressurization, and inspiratory cycling-off, were examined. The changes in tidal volumes in the D region based on the control settings were compared to assess the severity of pendelluft. RESULTS: The gas flow from the $\mathrm{D}$ region to the ND region was found to be essential in pendelluft, but the severity of this phenomenon was not always proportional to gas flows. The severity increased with the increase in the differences in pleural pressure levels between the ND and D regions, and it was amplified by the difference in lung mechanics between the ND and D regions. However, the ventilator settings had minimal effect on the severity of pendelluft. CONCLUSIONS: The pendelluft was affected by the heterogeneity of lung mechanics and pleural pressure. Furthermore, a minimal association was observed between the ventilator settings and the severity of pendelluft. Key words: pendelluft phenomenon; model lung; pleural pressure; ventilatory modes; triggering sensitivity; inspiratory pressurization; inspiratory cycling-off. [Respir Care 2021;66(1):1-10. (C) 2021 Daedalus Enterprises]
\end{abstract}

\section{Introduction}

Pendelluft phenomenon is a recently reported concept in mechanically ventilated subjects with ARDS who have preserved spontaneous breathing effort. ${ }^{1}$ This phenomenon is

Drs Enokidani, Uchiyama, Yoshida, Abe, Koyama, and Fujino are affiliated with the Department of Anesthesiology and Intensive Care Medicine, Osaka University Graduate School of Medicine, Suita, Japan. Dr Yamashita is affiliated with the Department of Anesthesiology, Osaka Women's and Children's Hospital, Izumi, Japan.

The authors have disclosed no conflicts of interest.

Supplementary material related to this paper is available at http://www. rcjournal.com. defined as gas movement inside the lung involving the displacement of gas from a more recruited nondependent (ND) lung region to a less recruited dependent (D) lung region without minimal changes in the tidal volume $\left(\mathrm{V}_{\mathrm{T}}\right)$ of the ventilator. Moreover, pendelluft causes overinflation in the $\mathrm{D}$ lung region and collapse in the ND lung region, which may cause lung injury. Therefore, if possible, appropriate ventilatory settings must be used to reduce the occurrence of

\footnotetext{
Correspondence: Akinori Uchiyama MD PhD, Department of Anesthesiology and Intensive Care Medicine, Osaka University Graduate School of Medicine, Yamadaoka 2-15, Suita, Osaka Pref., 565-0871, Japan. E-mail: auchiyama@hp-icu.med.osaka-u.ac.jp.
}

DOI: $10.4187 /$ respcare.07880 
pendelluft. However, because pendelluft develops without changes in the $V_{T}$ of the ventilator, it is difficult to recognize

\section{See the Related Editorial on Page 170}

via ordinary monitoring during mechanical ventilation. Porcine models of ARDS were used in the first report about pendelluft. Because a similar simulated condition of pendelluft is challenging to establish using animal models, the effects of ventilatory settings on this phenomenon cannot be accurately assessed. Thus, this study aimed to establish a lung model for pendelluft and to examine factors, including ventilatory settings, that are more likely to affect the occurrence of this phenomenon. Moreover, the effects of various ventilatory settings on pendelluft phenomenon were examined to identify which settings can minimize its severity.

\section{Methods}

\section{Simulation of Pendelluft Using a Lung Model System}

As shown in Figure 1, 2 sets of twin-bellows-type training test lung (TTL) models (Dual Adult Training Test Lung, Michigan Instruments, Grand Rapids, Michigan) were used in the experiment, and these models were similar to those utilized in the simulation of spontaneous breathing in a previous study. ${ }^{2}$ In this model, one set of bellows simulated the diaphragm, which established a negative pleural pressure $\left(\mathrm{P}_{\mathrm{pl}}\right)$, and the other model simulated the lung region. The diaphragm and lung bellows were connected to

\section{QUICK LOOK}

\section{Current knowledge}

The pendelluft phenomenon is defined as the displacement of gas from a more recruited nondependent lung region to a less recruited dependent lung region, and this condition may cause lung injury. Ventilatory settings that can minimize the severity of pendelluft are not fully elucidated.

\section{What this paper contributes to our knowledge}

Pendelluft was affected by the heterogeneity of lung mechanics and negative pleural pressures of the nondependent and dependent lung regions. A minimal association was observed between ventilatory mode, trigger sensitivity, inspiratory pressurization, and inspiratory cycle as well as the severity of the pendelluft.

each other to simulate spontaneous breathing. One TTL model represented the ND lung region, and the other represented the D lung region. The 2 lung region bellows were connected to each other via the artificial bronchus and trachea.

The PB840 (Covidien, Boulder, Colorado) ventilated both lung region bellows via an endotracheal tube with a 7.5-mm internal diameter, a heat-and-moisture exchanger (DAR Hygrobac S, Covidien), and a standard ventilator circuit (DAR adult ordinary circuit, Covidien). The flow was measured using 3 pneumotachographs (model 4700; Hans

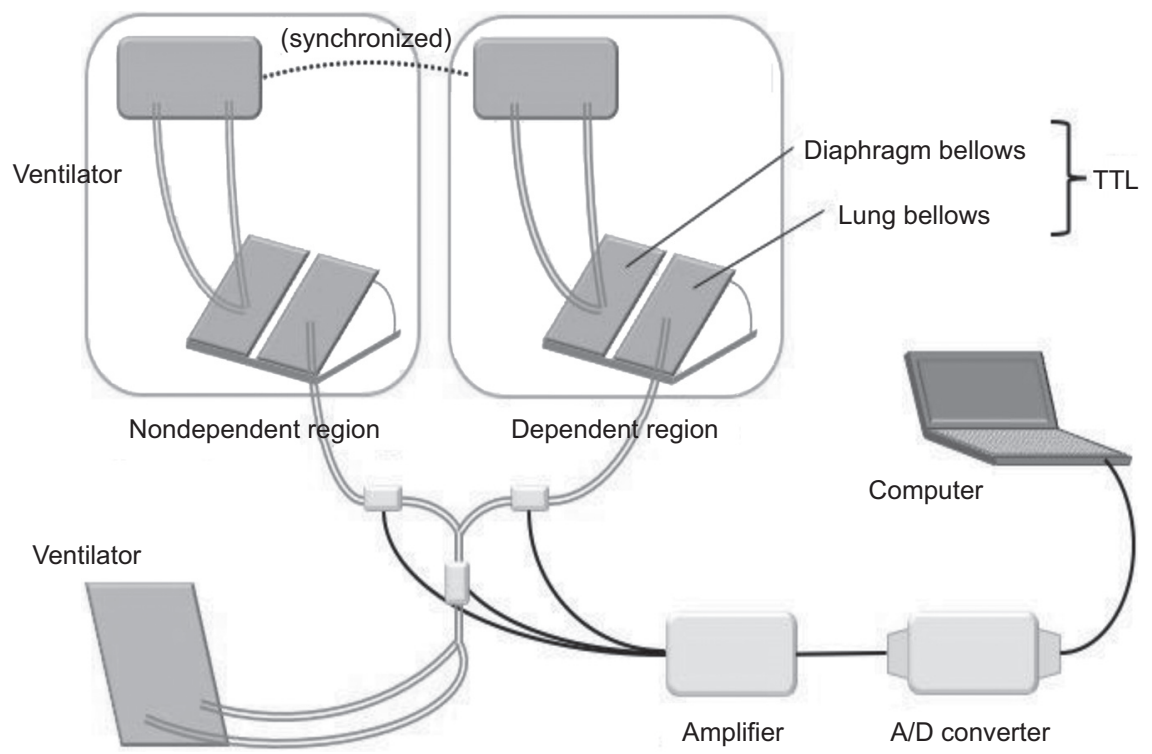

Fig. 1. Lung model setup. We developed the following devices: 2 pairs of twin-bellows-type (TTL) models, 2 AVEA ventilators, one PB 840 ventilator, 1 amplifier, 1 analog/digital converter (AVD), and a personal computer. Each pair of TTL and AVEA represented the nondependent and dependent lung regions, respectively. 
Table 1. Simulation Protocol for Negative Pleural Pressure

\begin{tabular}{|c|c|c|c|c|}
\hline & $\begin{array}{c}\text { Inspiratory } \\
\text { Pressure, } \\
\mathrm{cm} \mathrm{H}_{2} \mathrm{O}\end{array}$ & $\begin{array}{l}\text { Inspiratory } \\
\text { Time, s }\end{array}$ & Pressurization & $\begin{array}{c}\text { PEEP, cm } \\
\mathrm{H}_{2} \mathrm{O}\end{array}$ \\
\hline None & NA & NA & NA & NA \\
\hline Weak & 10 & 0.6 & Slow & 10 \\
\hline Medium & 20 & 0.75 & Medium & 10 \\
\hline Strong & 30 & 0.9 & Fast & 10 \\
\hline $\begin{array}{c}\text { Extremely } \\
\text { strong }\end{array}$ & 40 & 1.05 & Fast & 10 \\
\hline \multicolumn{5}{|c|}{$\begin{array}{l}\text { To simulate negative pleural pressure, AVEA ventilators were used based on this protocol. A } \\
\text { larger inspiratory pressure in an AVEA represented a higher level of negative pleural pressure. } \\
\text { When we chose "None," AVEA was kept on stand-by, thereby representing the condition with } \\
\text { no breathing effort. } \\
\text { NA = not applicable }\end{array}$} \\
\hline
\end{tabular}

Rudolph, Kansas City, Missouri), which were placed at the proximal end of the endotracheal tube and the points between the artificial bronchus and the lung region bellows, as well as differential pressure transducers (TP602T; Nihon Kohden, Tokyo, Japan), and amplifiers (AR601G; Nihon Kohden,). Moreover, airway pressure was measured using a differential pressure transducer (TP603T) and an amplifier (AR601G) at the proximal end of the endotracheal tube. The signals from the amplifiers were recorded at a frequency of $100 \mathrm{~Hz}$ and were analyzed using a data acquisition system (WINDAQ, Dataq Instruments, Akron, Ohio).

The diaphragm bellows of the TTLs, for which the compliance was set at $20 \mathrm{~mL} / \mathrm{cm} \mathrm{H}_{2} \mathrm{O}$, were ventilated with 2 Avea ventilators (CareFusion, San Diego, California), which were connected by wire and synchronized with a ventilatory frequency of 20 breaths/min. The negative $P_{p l}$ levels were regulated using the ventilatory settings of the Avea ventilators. We simulated pendelluft phenomenon by applying 2 different levels of negative $\mathrm{P}_{\mathrm{pl}}$ to the ND and $\mathrm{D}$ bellows. The ventilatory settings of the Avea ventilators were changed to establish 5 levels of negative $\mathrm{P}_{\mathrm{pl}}$, as shown in Table 1. The level of "None" simulated no breathing effort without using diaphragm bellows by stopping the Avea ventilators. The compliance and airway resistance of the lung region bellows were set according to previous reports. ${ }^{3-5} \mathrm{We}$ established 2 patterns of regional lung mechanics: the mechanics referred to as normal included a compliance of $40 \mathrm{~mL} / \mathrm{cm} \mathrm{H}_{2} \mathrm{O}$ and a resistance of $5 \mathrm{~cm} \mathrm{H}_{2} \mathrm{O} / \mathrm{L} / \mathrm{s}$, and the pattern referred to as damaged included a compliance of 10 $\mathrm{mL} / \mathrm{cm} \mathrm{H}_{2} \mathrm{O}$ and a resistance of $20 \mathrm{~cm} \mathrm{H}_{2} \mathrm{O} / \mathrm{L} / \mathrm{s}$.

To validate the effects of $\mathrm{P}_{\mathrm{pl}}$ and lung mechanics on pendelluft, 4 different negative $\mathrm{P}_{\mathrm{pl}}$ levels in the $\mathrm{D}$ lung region (Table 1) were applied with a weak negative $\mathrm{P}_{\mathrm{pl}}$ in the ND lung region under 2 different mechanics (ie, normal and damaged) in the $\mathrm{D}$ region. The experimental setting, according to the previous report, ${ }^{1}$ was decided based on the following reasons: the pathological abnormality of the D region is more prominent than that of the ND region in the lungs with ARDS; spontaneous effort was associated with a large vertical gradient of $\mathrm{P}_{\mathrm{pl}}$ swings from the ND to the $\mathrm{D}$ regions in the injured lung; and pendelluft resulted from the development of a more negative swing in $\mathrm{P}_{\mathrm{pl}}$ in the $\mathrm{D}$ lung region than that in the ND lung region. The settings ventilating the lung model were continuous mandatory ventilation with pressure control ventilation, ventilatory frequency of 15 breaths/min, inspiratory pressure of $12 \mathrm{~cm} \mathrm{H}_{2} \mathrm{O}$, inspiratory time of $1.2 \mathrm{~s}$, inspiratory pressurization of $50 \%$, trigger sensitivity of $-2 \mathrm{~cm} \mathrm{H}_{2} \mathrm{O}$, and PEEP of $15 \mathrm{~cm} \mathrm{H}_{2} \mathrm{O}$. PEEP set at $15 \mathrm{~cm} \mathrm{H}_{2} \mathrm{O}$ is higher than its usual range, but it was necessary to hold a certain amount of residual gas volume in the lung region to examine gas movements from one lung region to another. The normal physiologic end-expiratory $\mathrm{P}_{\mathrm{pl}}$ is negative to maintain the residual lung volume. However, the pressure around the lung model was atmospheric, so we used high PEEP to maintain residual volume.

Ventilatory volumes were calculated via the integration of the flow signals at 3 parts, namely, the D lung region, the ND lung region, and the whole lung. Based on previous reports, pendelluft is defined as a ventilatory volume change caused by the use of a negative $\mathrm{P}_{\mathrm{pl}}$ level. ${ }^{1}$ The incremental increase in $\mathrm{V}_{\mathrm{T}}$ in the $\mathrm{D}$ lung region with no breathing effort was used to assess the extent of pendelluft. Representative waveforms acquired from the model are shown in Figure 2. Moreover, gas inflow to the D lung region and a corresponding outflow from the ND lung region was assessed during early inspiration (red band in Fig. 2). The gas movement in this study, which is considered as the "to" gas movement, was consistent with that in a previous report. ${ }^{1}$ Furthermore, we observed a gas inflow to the ND lung region and a corresponding outflow from the D lung region during end inspiration (blue band in Fig. 2); this is considered as the "fro" gas movement, which is the counterpart of the "to" gas movement.

\section{Effects of Ventilatory Settings on Pendelluft}

To identify various ventilatory settings that can minimize the severity of pendelluft, we examined the effects of mode, trigger sensitivity, inspiratory pressurization, and cycling-off criteria. The changes in $\mathrm{V}_{\mathrm{T}}$ in the $\mathrm{D}$ lung region based on the control setting were used to assess the effects of ventilatory settings on the severity of pendelluft.

The effects of ventilatory settings on pendelluft were examined under fixed settings: normal lung mechanics and weak negative $\mathrm{P}_{\mathrm{pl}}$ in the ND lung region, and damaged lung mechanics and extremely strong negative $\mathrm{P}_{\mathrm{pl}}$ in the $\mathrm{D}$ lung region. The 2 Avea ventilators were connected by a wire and synchronized with a ventilatory frequency of 20 breaths/min. 


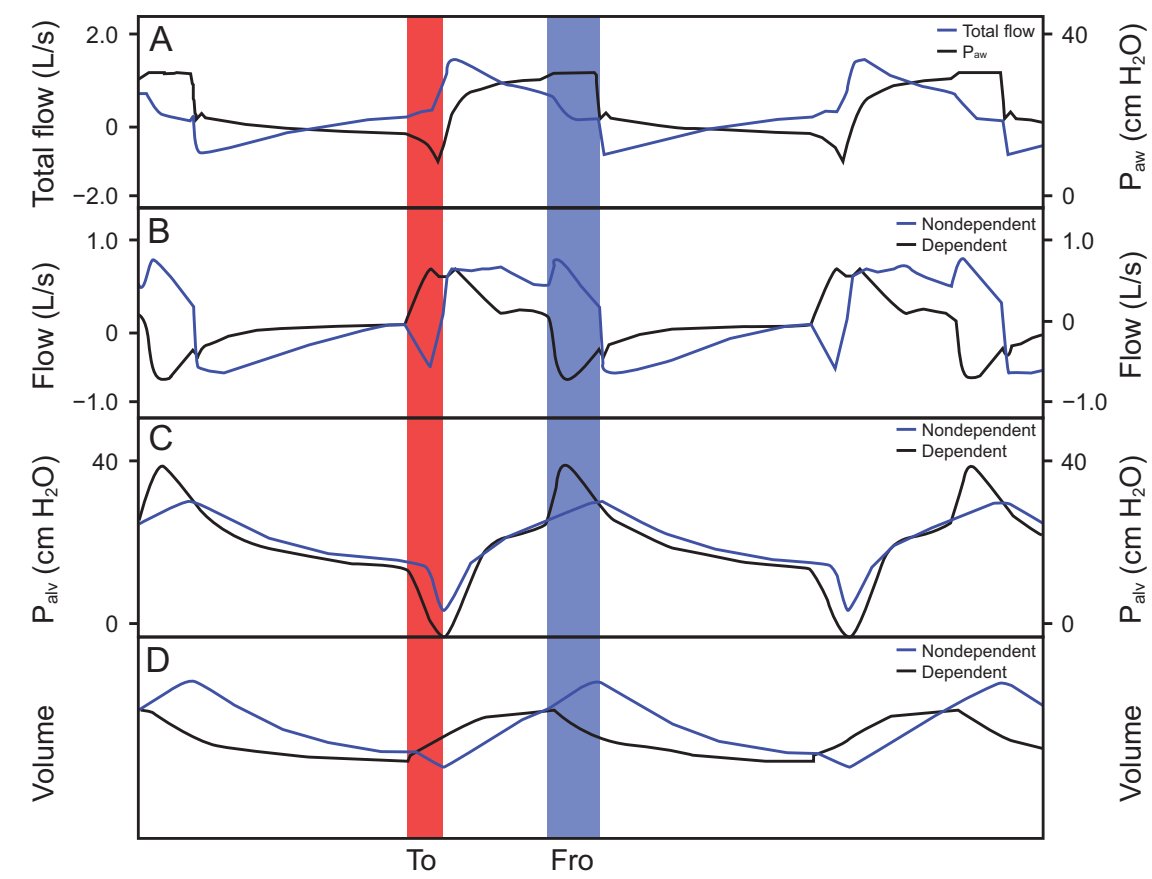

Fig. 2. Representative waveforms of pendelluft phenomenon. The representative waveforms acquired in a condition with normal lung mechanics and weak negative pleural pressure $\left(P_{p l}\right)$ levels in the nondependent lung region and damaged lung mechanics and strong negative $P_{p l}$ level in the dependent lung region. $A$ : The airway pressure $\left(P_{a w}\right)$ and the flow of the ventilator. The gas flow in each region is depicted in $(B)$, where upward and downward waveforms represent the inflow and outflow to the region, respectively. An upward waveform (ie, inflow) in the dependent lung region and the corresponding downward waveform (ie, outflow) in the nondependent lung region (red) exhibit gas movement from the nondependent to the dependent lung region, which is defined as the "to" flow. By contrast, an upward waveform in the nondependent lung region and the corresponding downward waveform in the dependent lung region (blue) exhibit gas movement from the dependent to the nondependent lung region, which is defined as the "fro" flow. C: Alveolar pressures $\left(P_{\text {alv }}\right)$ of the dependent and nondependent lung regions. D: Volumes of the dependent and nondependent lung regions.

First, we compared 2 ventilatory modes to examine the effects of ventilatory modes on pendelluft phenomenon: (1) as the control test, continuous mandatory ventilation with pressure control ventilation of $12 \mathrm{~cm} \mathrm{H}_{2} \mathrm{O}$, inspiratory time of $1.2 \mathrm{~s}$, trigger sensitivity of $-2 \mathrm{~cm} \mathrm{H}_{2} \mathrm{O}$, inspiratory pressurization of 50\%, and PEEP of $15 \mathrm{~cm} \mathrm{H}_{2} \mathrm{O}$; and (2) continuous mandatory ventilation with volume control ventilation with a $\mathrm{V}_{\mathrm{T}}$ of $600 \mathrm{~mL} / \mathrm{kg}$, which is similar to other experiments, inspiratory time of $1.2 \mathrm{~s}$, trigger sensitivity of $-2 \mathrm{~cm}$ $\mathrm{H}_{2} \mathrm{O}$, constant inspiratory flow, and PEEP of $15 \mathrm{~cm} \mathrm{H}_{2} \mathrm{O}$.

Second, 5 inspiratory triggering settings (flow-triggering sensitivity of 1 and $3 \mathrm{~L} / \mathrm{min}$ [f-1, f-2] and trigger sensitivity settings of 2 [control], 4 , and $6 \mathrm{~cm} \mathrm{H}_{2} \mathrm{O}$ [p-2, p-4, and p-6], respectively) were compared to examine the effects of inspiratory triggering sensitivity on pendelluft. The other settings were continuous mandatory ventilation with pressure control ventilation, ventilatory frequency of 15 breaths/min, inspiratory pressure of $12 \mathrm{~cm} \mathrm{H}_{2} \mathrm{O}$, inspiratory time of $1.2 \mathrm{~s}$, inspiratory pressurization of 50\%, and PEEP of $15 \mathrm{~cm} \mathrm{H}_{2} \mathrm{O}$.

Third, 5 inspiratory pressurization settings $(1 \%, 25 \%$, $50 \%$ [control], $75 \%$, and $100 \%$ ) were compared to examine the effects this variable on pendelluft. The other settings were continuous mandatory ventilation with pressure control ventilation, ventilatory frequency of 15 breaths/min, inspiratory pressure of $12 \mathrm{~cm} \mathrm{H}_{2} \mathrm{O}$, inspiratory time of $1.2 \mathrm{~s}$, trigger sensitivity of $-2 \mathrm{~cm} \mathrm{H}_{2} \mathrm{O}$, and PEEP of $15 \mathrm{~cm} \mathrm{H}_{2} \mathrm{O}$.

Fourth, the effects of cycling-off criteria on pendelluft phenomenon were examined using 6 inspiratory time settings $(0.6,0.8,1.0,1.2,1.4$, and $1.6 \mathrm{~s})$, and the other settings were continuous mandatory ventilation with pressure control ventilation, ventilatory frequency of 15 breaths/min, inspiratory pressure of $12 \mathrm{~cm} \mathrm{H}_{2} \mathrm{O}$, trigger sensitivity of -2 $\mathrm{cm} \mathrm{H}_{2} \mathrm{O}$, and PEEP of $15 \mathrm{~cm} \mathrm{H}_{2} \mathrm{O}$. Moreover, the effects of 3 cycling-off criteria of pressure support ventilation of 12 $\mathrm{cm} \mathrm{H}_{2} \mathrm{O}$, trigger sensitivity of $-2 \mathrm{~cm} \mathrm{H}_{2} \mathrm{O}$, and PEEP of 15 $\mathrm{cm} \mathrm{H}_{2} \mathrm{O}$ were examined.

\section{Statistical Analysis}

We performed a one-way analysis of variance (ANOVA). If a significant result was obtained, a post hoc analysis was performed by comparing data with those of the control settings using the Dunnett test. A $P$ value $<.01$ was considered statistically significant. Acquired data distribution ranges were relatively lower in the bench study than those in other studies. A more stringent $P$ value was 

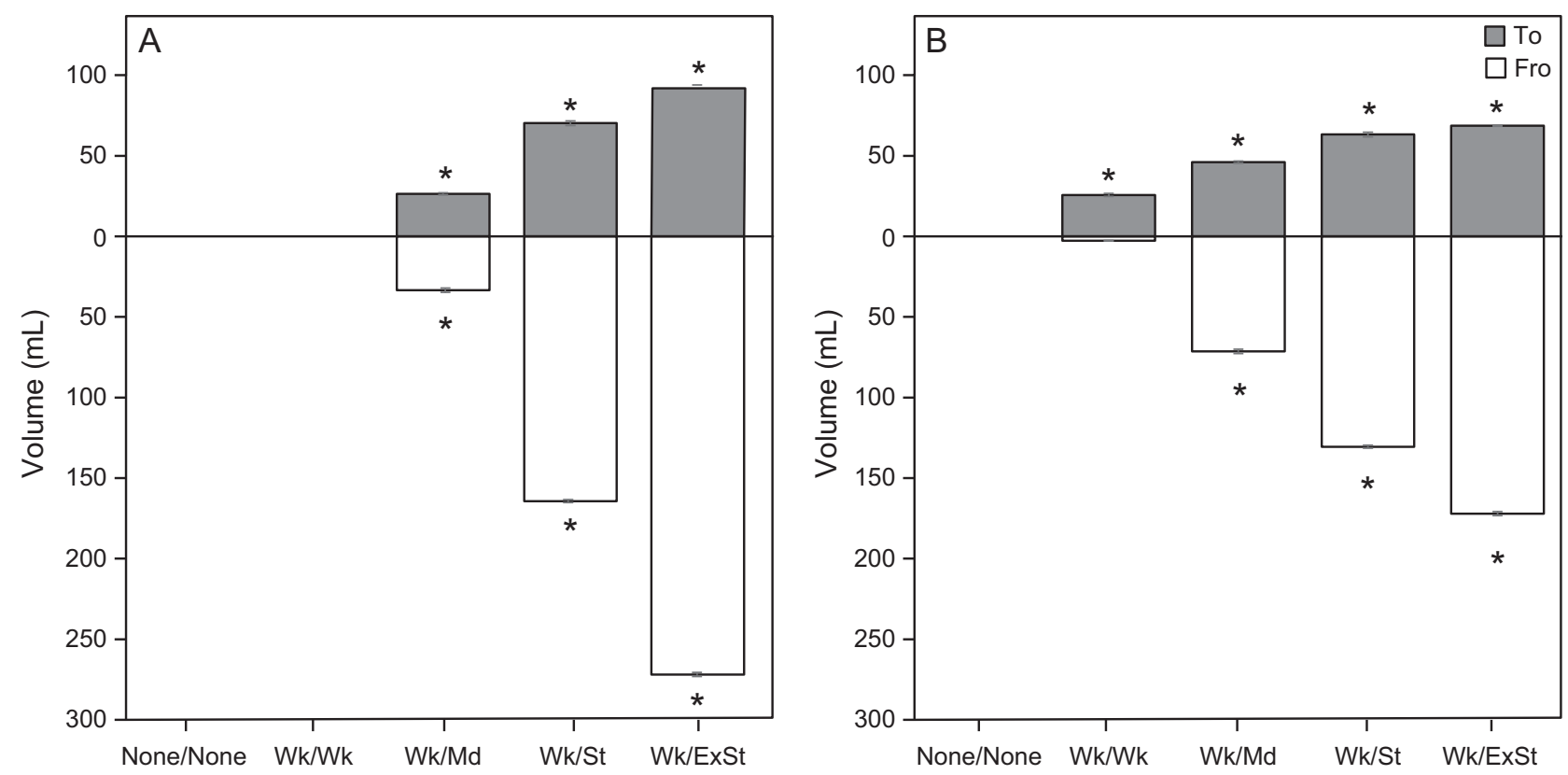

Fig. 3. Integrated volumes of the "to" and "fro" flows with 5 different combinations of pleural pressures under 2 regional lung mechanics settings. A: Normal lung mechanics of both the nondependent and dependent lung regions. B: Normal lung mechanics of the nondependent lung region and damaged lung mechanics of the dependent lung regions. Wk $=$ weak, Md $=$ Medium, St $=$ Strong, and ExSt $=$ Extremely strong pleural pressures (refer to Table 1). *Significantly different from the values without spontaneous breathing efforts (None/None) $(P<.01)$.

applied to reach meaningful conclusions from the bench study, similar to our previous study. ${ }^{6}$

\section{Results}

\section{Effects of Regional Lung Mechanics and Strength of Negative $\mathbf{P}_{\mathrm{pl}}$ on Pendelluft}

The integrated volumes of the "to" and "fro" flows with 5 different combinations of $\mathrm{P}_{\mathrm{pl}}$ under the 2 regional lung mechanic settings are shown in Figure 3. Although the volumes of the "to" and "fro" flows were not observed in the absence of spontaneous breathing effort, these volumes were observed in cases of different $\mathrm{P}_{\mathrm{pl}}$ on the ND and D lung regions. With a similar weak negative $\mathrm{P}_{\mathrm{pl}}$ in the ND and D lung regions, the volumes of the "to" and "fro" flows were not observed in cases involving similar regional lung mechanics in the ND and D lung regions. However, these volumes were noted in cases involving different regional lung mechanics in the ND and D lung regions.

The $\mathrm{V}_{\mathrm{T}}$ values of the whole lung and the ND and $\mathrm{D}$ lung regions with 5 different combinations of $\mathrm{P}_{\mathrm{pl}}$ under the 2 regional lung mechanic settings are shown in Figure 4. The total and regional $\mathrm{V}_{\mathrm{T}}$ were affected by the $\mathrm{P}_{\mathrm{pl}}$. As the $\mathrm{P}_{\mathrm{pl}}$ in the $\mathrm{D}$ lung region decreased, the $\mathrm{V}_{\mathrm{T}}$ of the $\mathrm{D}$ and ND lung regions increased, even if the $\mathrm{P}_{\mathrm{pl}}$ in the ND lung region did not change. The total $\mathrm{V}_{\mathrm{T}}$ was not significantly different from that without spontaneous breathing effort, except in the case of an extremely strong $\mathrm{P}_{\mathrm{pl}}$ in the D lung region. In particular, damaged lung mechanics in the $\mathrm{D}$ lung region resulted in larger pendelluft volumes even in the small basal $\mathrm{V}_{\mathrm{T}}$ of the $\mathrm{D}$ lung region.

In a previous study, the volumes of pendelluft (ie, pendelluft volumes) were defined as the regional $\mathrm{V}_{\mathrm{T}}$ changes in the $\mathrm{D}$ lung region in the absence of spontaneous breathing effort (None/None). ${ }^{1}$ The relationship between the changes in the total $\mathrm{V}_{\mathrm{T}}$ and pendelluft volumes with 4 different combinations of $\mathrm{P}_{\mathrm{pl}}$ are shown in Figure 5. The pendelluft volumes were larger than the changes in total $\mathrm{V}_{\mathrm{T}}$. Significant pendelluft volumes were observed in the $a b-$ sence of changes in total $\mathrm{V}_{\mathrm{T}}$. Furthermore, the relationship between the integrated volumes of the "to" or "fro" flows and pendelluft volumes with 4 different combinations of $\mathrm{P}_{\mathrm{pl}}$ are depicted in Figure 5. The pendelluft volumes were significantly larger than the integrated volumes of the "to" flow, and they were extremely similar to those of the "fro" flow. When the gas flows between the ND and D lung regions, significant pendelluft volumes could develop.

\section{Relationship Between Pendelluft and Ventilatory Settings}

As shown in Figure 6, the integrated volumes of the "to" and "fro" flows of volume control ventilation were lower than those of pressure control ventilation. With a similar total $\mathrm{V}_{\mathrm{T}}$ condition, $\mathrm{V}_{\mathrm{T}}$ in the $\mathrm{D}$ lung region of volume control ventilation did not differ from that of pressure control ventilation. This result indicated that the ventilatory mode had minimal effect on pendelluft. 

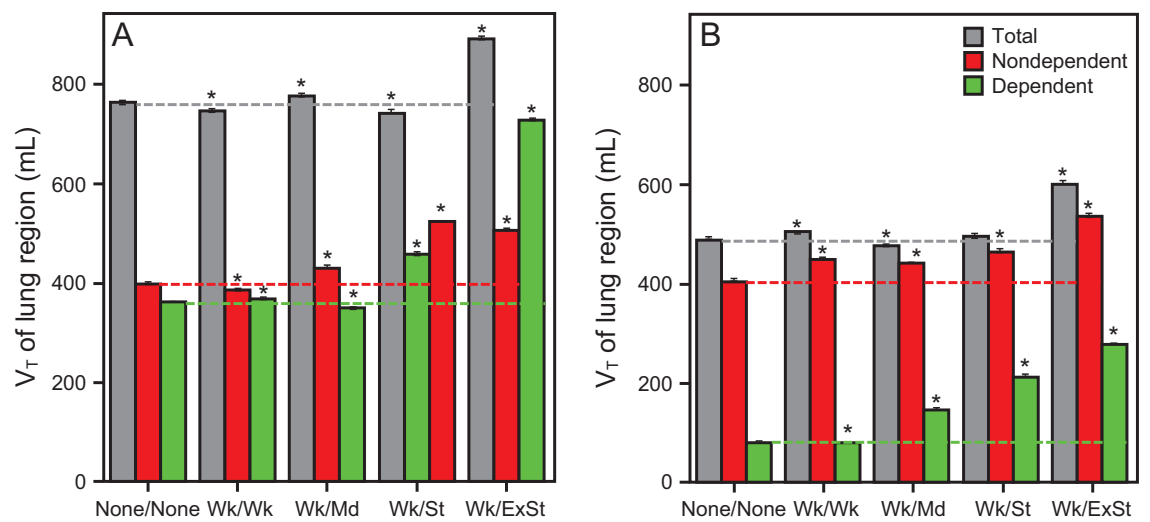

Fig. 4. Tidal volumes of the whole, nondependent, and dependent lung regions with 5 different combinations of pleural pressures under 2 regional lung mechanic settings. A: Normal lung mechanics of both the nondependent and dependent lung regions. B: Normal lung mechanics of the nondependent lung region and damaged lung mechanics of the dependent lung region. Dashed lines indicate the levels of tidal volumes without spontaneous breathing efforts (None/None). $\mathrm{Wk}=$ weak, Md = Medium, St $=$ Strong, and ExSt $=$ Extremely strong pleural pressures (refer to Table 1). *Significantly different from the values without spontaneous breathing efforts (None/None) $(P<.01)$.
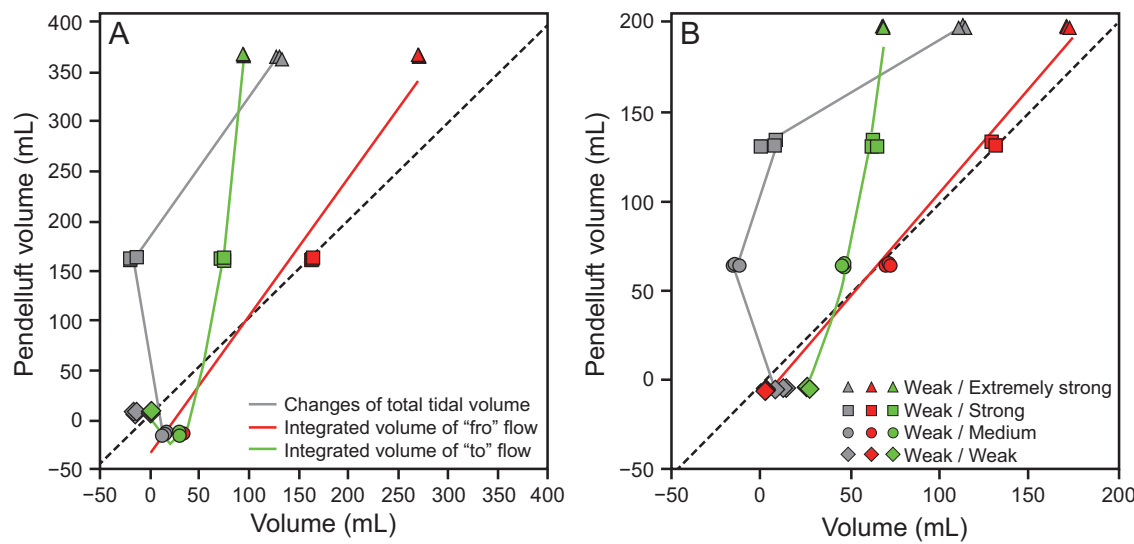

Fig. 5. Relationship between the integrated volumes of the "to" or "fro" flow or changes in total tidal volume and pendelluft volumes with 4 different combinations of pleural pressures under 2 regional lung mechanic settings. A: Normal lung mechanics of both the nondependent and dependent lung regions. B: Normal lung mechanics of the nondependent lung region and damaged lung mechanics of the dependent lung region. The pendelluft volumes were defined as the differences in tidal volumes based on the values without spontaneous breathing efforts (None/None) in the dependent lung region.

As shown in Figure 7, the integrated volumes of the "to" and "fro" flows in $\mathrm{f}-1, \mathrm{f}-3, \mathrm{p}-4$, and $\mathrm{p}-6$ were different from those in $\mathrm{p}-2$ (control). The inspiratory triggering settings affected the total $\mathrm{V}_{\mathrm{T}}$ and $\mathrm{V}_{\mathrm{T}}$ in the ND region. However, the triggering settings did not affect $\mathrm{V}_{\mathrm{T}}$ in the $\mathrm{D}$ region. This result indicated that inspiratory triggering settings had minimal effect on pendelluft.

As shown in Figure 8, the integrated volumes of the "to" flow at pressurization settings of $1 \%, 25 \%, 75 \%$, and $100 \%$ were different from that at $50 \%$ (control). Meanwhile, the integrated volume of the "fro" flow at a pressurization setting of $1 \%$ was lower than that at $50 \%$ (control). Although inspiratory pressurization settings affected the total $\mathrm{V}_{\mathrm{T}}$ and $\mathrm{V}_{\mathrm{T}}$ in the ND lung region, the $\mathrm{V}_{\mathrm{T}}$ in the $\mathrm{D}$ lung region at a pressurization setting of $1 \%$ was slightly higher than that at $50 \%$. The $\mathrm{V}_{\mathrm{T}}$ in the $\mathrm{D}$ lung region at other pressurization settings did not differ from that at 50\%. This result indicated that inspiratory pressurization settings had minimal effect on pendelluft phenomenon.

As shown in Figure 9, the integrated volumes of the "to" flow in all the cycling-off criteria were different from those at a inspiratory time of $1.2 \mathrm{~s}$ (control). The integrated volumes of the "fro" flow at all cycling-off criteria except at a inspiratory time of $1.4 \mathrm{~s}$ were different from those at $1.2 \mathrm{~s}$ (control). The "fro" flow disappeared at a inspiratory time of $0.6 \mathrm{~s}$ and at a cycling-off setting of $40 \%$ for pressure support ventilation. However, there were no changes observed in pendelluft. Although the inspiratory cycling-off settings affected the "to" and "fro" flow volumes, inspiratory cycling-off criteria had minimal changes influence on $\mathrm{V}_{\mathrm{T}}$ in the $\mathrm{D}$ lung region. This result indicated that inspiratory cycling-off criteria had minimal effect on pendelluft. In 


\section{Ventilatory Settings and Pendelluft Phenomenon}
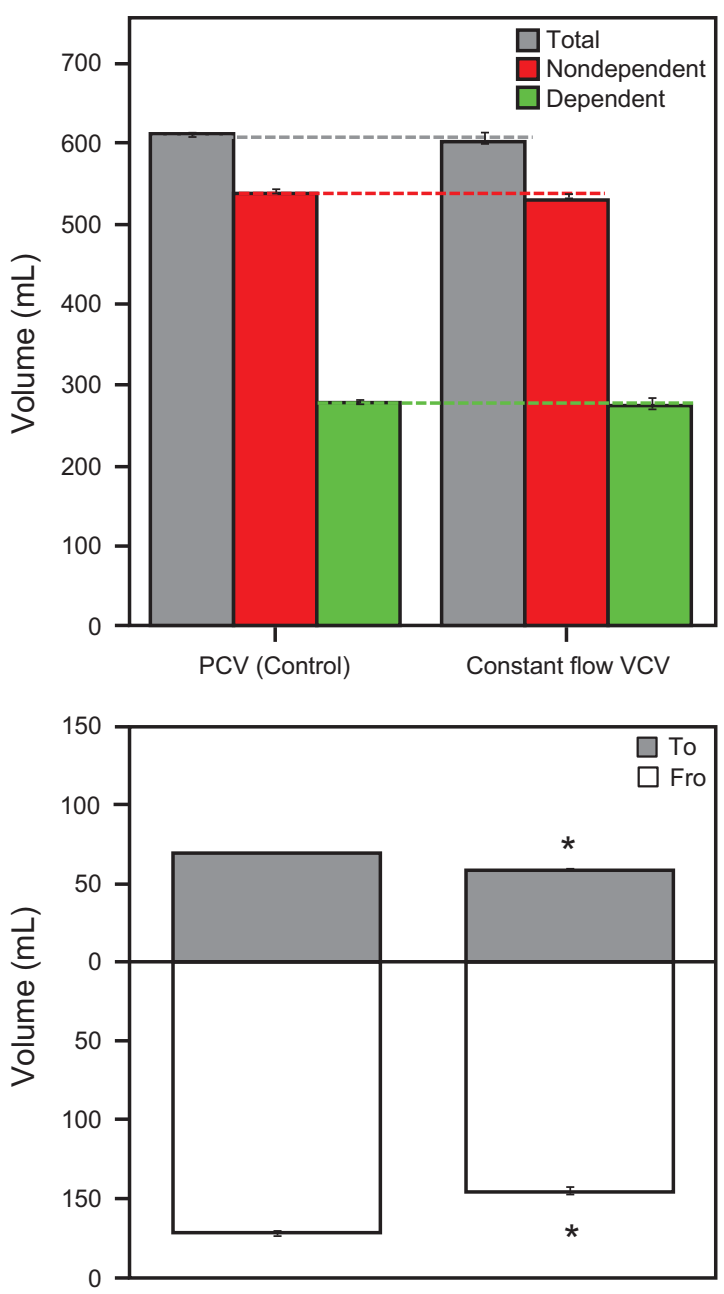

Fig. 6. Tidal volumes of the whole, nondependent, and dependent lung regions and the integrated volumes of the "to" and "fro" flows under volume control ventilation (VCV) mode and pressure control ventilation (PCV) mode. The VCV mode was set at a tidal volume and inspiratory time similar to those of PCV. The PCV mode was used as a control. The dashed lines indicate the levels of the tidal volumes of PCV mode as a control. *Significantly different from the values of PCV mode $(P<.01)$.

contrast, the total $\mathrm{V}_{\mathrm{T}}$ and $\mathrm{V}_{\mathrm{T}}$ in the ND region were affected by the inspiratory cycling-off criteria.

\section{Discussion}

This study showed that a lung model for pendelluft could be established. Although the gas flow from the ND lung region to the D lung region was essential in pendelluft, the severity of pendelluft was not always proportional to gas flow levels. Moreover, severity increased with the increase in the differences in negative $\mathrm{P}_{\mathrm{pl}}$ levels between the ND and D lung regions. In addition, the severity of pendelluft was amplified by the difference in lung mechanics between the ND and D lung regions. However,
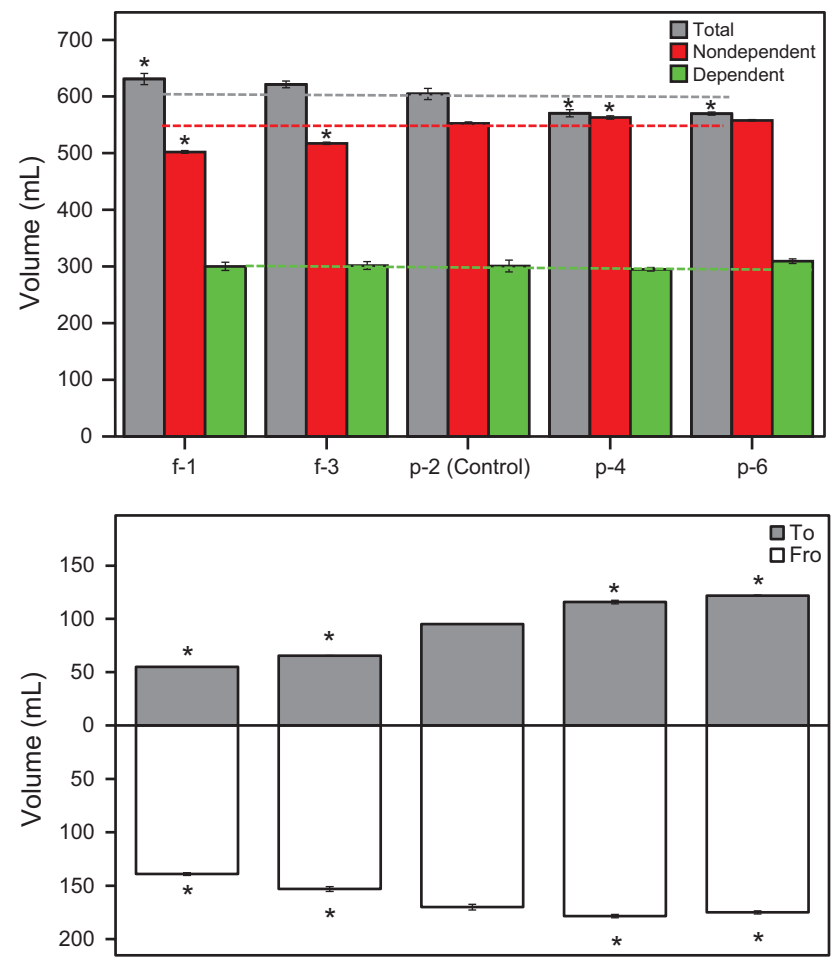

Fig. 7. Tidal volumes of the whole, nondependent, and dependent lung regions and the integrated volumes of the "to" and "fro" flows under 5 inspiratory triggering settings. Flow-triggering of $1 \mathrm{~L} / \mathrm{min}$ and $3 \mathrm{~L} / \mathrm{min}$ ( $\mathrm{f}-1$ and $\mathrm{f}-3$ ) and pressure-triggering of 2, 4, and $6 \mathrm{~cm}$ $\mathrm{H}_{2} \mathrm{O}(\mathrm{p}-2, \mathrm{p}-4$, and $\mathrm{p}-6)$ were compared. The values of $\mathrm{p}-2$ were used as a control. The dashed lines indicate the levels of tidal volumes of $\mathrm{p}-2$ used as a control. *Significantly different from the values of $\mathrm{p}-2(P<.01)$.

the ventilator settings (eg, ventilation mode, trigger sensitivity, inspiratory pressurization, and inspiratory cyclingoff) had minimal effects on the degree of pendelluft.

The differences in negative $\mathrm{P}_{\mathrm{pl}}$ between the ND and $\mathrm{D}$ lung regions induce the "to" and "fro" flows between the lung regions. Pendelluft is characterized by the "to" and "fro" flows. As the differences in negative $\mathrm{P}_{\mathrm{pl}}$ increased, the volumes of the "to" and "fro" flows increased. As the volumes of the "to" flow increased, the increase of $\mathrm{V}_{\mathrm{T}}$ in the $\mathrm{D}$ lung region, which represented the severity of pendelluft phenomenon, increased. However, the increase of $\mathrm{V}_{\mathrm{T}}$ in the $\mathrm{D}$ lung region was not always proportional to the volume of the "to" flow. Although the increase of $\mathrm{V}_{\mathrm{T}}$ in the $\mathrm{D}$ lung region might be relatively parallel to the volumes of the "fro" flow in the first experiment, it was not applicable to the experiments with various inspiratory cycling-off criteria. Thus, in pendelluft, the existence of the "to" flows is more essential than that of the "fro" flow in all experiment settings.

A mathematical model of developing the "to" gas movement from the ND lung region to the $\mathrm{D}$ lung region is 

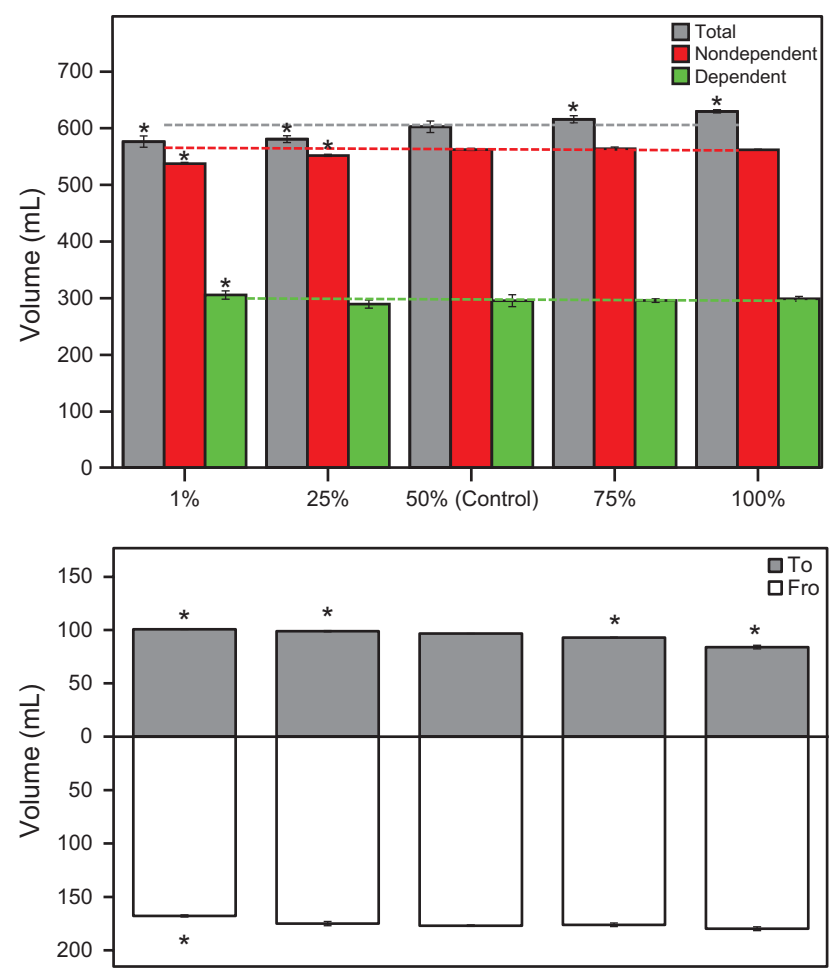

Fig. 8. Tidal volumes of the whole, nondependent, and dependent lung regions and the integrated volumes of the "to" and "fro" flows under 5 inspiratory pressurization settings. Five inspiratory pressurization settings from slow to rapid $(1 \%, 25 \%, 50 \%, 75 \%$, and $100 \%$ ) were compared. The values of $50 \%$ were used as a control. The dashed lines indicate the levels of tidal volumes of $50 \%$ used as a control. *Significantly different from the values of $50 \%(P<.01)$.

provided in the online supplement (see the supplementary materials at http://www.rcjournal.com). According to the model, the difference in $\mathrm{P}_{\mathrm{pl}}$ between the ND and D lung regions is the major factor for the development of the "to" flow, which can determine the severity of pendelluft. The normal lung mechanics in the $\mathrm{D}$ lung region cannot reduce the severity of pendelluft. This phenomenon, accompanied by strong inspiratory efforts, might easily occur in the early phase of lung injury.

\section{Effects of Ventilatory Settings on Pendelluft}

We examined whether some mechanical ventilator settings, such as ventilatory mode, inspiratory triggering sensitivities, and inspiratory pressurization, affected the severity of pendelluft. These variables were reported to affect ventilatory status. Pressure control ventilation compared with volume control ventilation at a similar inspiratory time resulted in a more efficient elimination of $\mathrm{CO}_{2}$ per breath. ${ }^{7}$ Pressure control ventilation may favor ventilation of units with slow time constants, thereby increasing the mean distribution time, which was defined as the time available for gas distribution and diffusion during inspiration. The triggering function is a key determinant of the efficacy of assisted mechanical ventilation. ${ }^{89}$ The ventilator must have a short response time to maintain optimal synchrony with inspiratory effort. The flow-triggering system is faster, and it is more effective than the pressure-triggering system in reducing a patient's work. ${ }^{8}$ Inspiratory pressurization settings can determine the speed at which airway pressure builds toward the preset maximum value. A faster increase in time must be selected during low-impedance conditions, whereas a slower increase in time should be selected during high-impedance conditions to prevent overshoot in airway pressure at the beginning of inspiration. $^{7}$ A higher inspiratory pressurization is also required in case of a higher inspiratory drive. ${ }^{1,10,11}$ Chiumello et $\mathrm{al}^{11,12}$ reported that both high and low pressure rise slopes increase discomfort and work of breathing. However, these variables had minimal effect on the severity of pendelluft. The adjustment of each setting resulted in a slight decrease in the degree of pendelluft. However, pendelluft remained even when a condition was optimized (ie, extremely short trigger delay or maximum pressurizing speed). These results indicate that no individual ventilator setting will effectively inhibit pendelluft.

Moreover, the effects of the differences in inspiratory cycling-off were examined. To improve synchrony during assisted pressure control ventilation, the spontaneous inspiratory time of the patients must match. The duration of inspiratory effort was set at $1.2 \mathrm{~s}$ in this study. Thus, the inspiratory time of the control setting was set at $1.2 \mathrm{~s}$. In addition, the inspiratory time of pressure control ventilation should be longer than the 3 time constants of the respiratory system to allow near complete (ie, $>95 \%$ ) lung filling, thereby maximizing the delivered $\mathrm{V}_{\mathrm{T}}$. Although inspiratory times of 1.0-1.5 s were required in ordinary adults with orotracheal intubation, inspiratory times as short as $0.8-1.0 \mathrm{~s}$ are recommended in patients with reduced lung compliance. ${ }^{7}$ Therefore, the various inspiratory times of pressure control ventilation were examined. Moreover, the cycling-off criteria of pressure support ventilation changed the inspiratory time and affected breathing pattern. ${ }^{12}$ The inspiratory times changed the "to" and "fro" flow volumes. The "fro" flow volumes almost disappeared in short inspiratory time settings. However, the $V_{T}$ of the D lung regions were similar. Moreover, the inspiratory cycling-off had minimal effects on pendelluft.

$\mathrm{P}_{\mathrm{pl}}$ difference between the ND and $\mathrm{D}$ lung regions is the main factor associated with the development of pendelluft. Our results indicate that the ventilator settings had little effect on the degree of pendelluft. However, inappropriate ventilator settings, such as insensitive triggering or inadequate inspiratory pressurization, develop stronger inspiratory efforts, which might enlarge the $\mathrm{P}_{\mathrm{pl}}$ difference between the ND and the D lung regions, leading to increased severity of pendelluft. Therefore, ventilator settings that are 

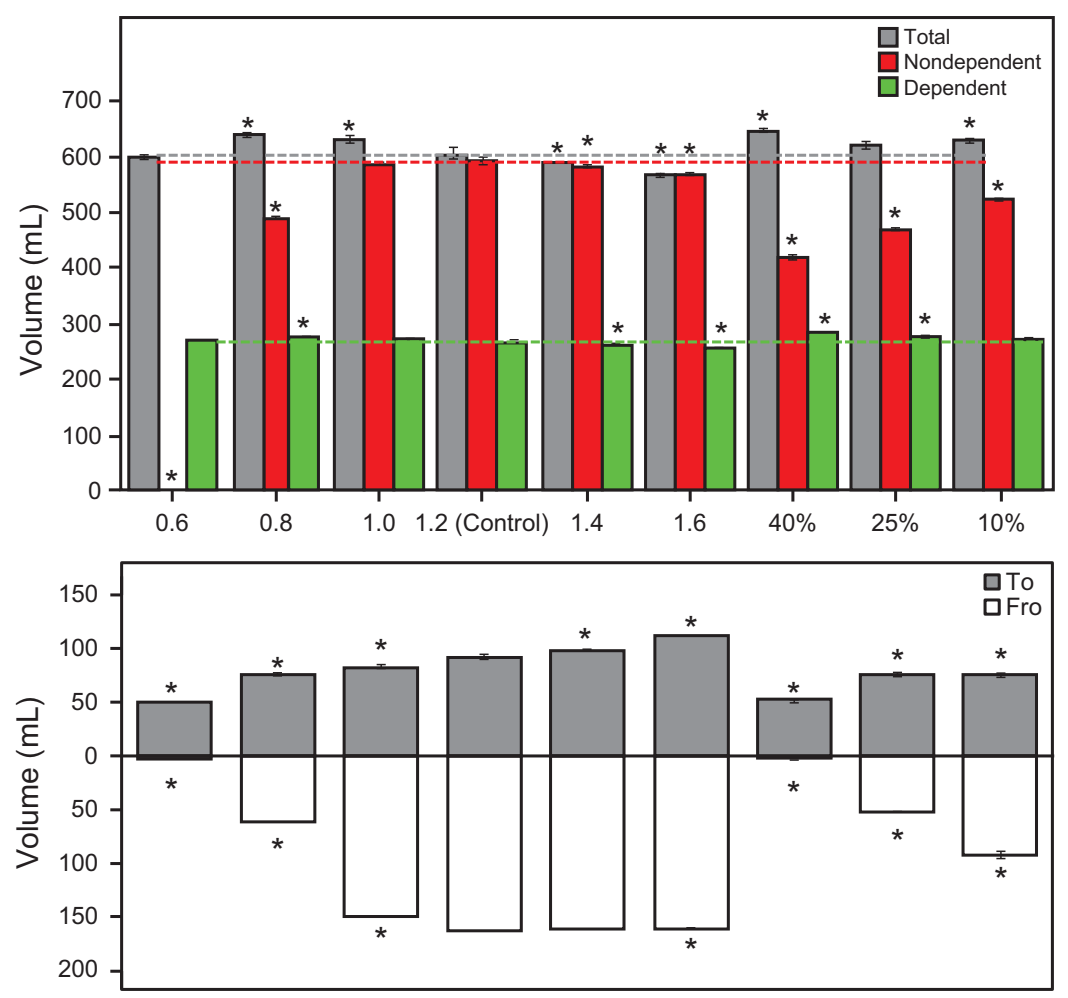

Fig. 9. Tidal volumes of the whole, nondependent, and dependent lung regions and the integrated volumes of the "to" and "fro" flows under 6 inspiratory time settings and 3 cycle-off settings of pressure support ventilation (PSV). Six inspiratory time settings from short to long (0.6, 0.8, $1.0,1.2,1.4$, and $1.6 \mathrm{~s}$ ) during continuous mandatory ventilation with pressure control ventilation (PCV) and 3 cycle of settings in continuous positive airway pressure (CPAP) mode with PSV (40\%, 25\%, and $10 \%$ of peak inspiratory flow) were compared. PSV was set at a peak airway pressure similar to that of PCV in CPAP + PSV mode. A inspiratory time setting of $1.2 \mathrm{~s}$ during continuous mandatory ventilation with PCV was used as a control. The dashed lines indicate the levels of tidal volumes at $1.2 \mathrm{~s}$ used as a control. *Significantly different from a inspiratory time setting of $1.2 \mathrm{~s}(P<.01)$.

more suitable for a patient's respiration might be an important factor in preventing ventilator-induced lung injury.

\section{Limitations}

The actual pendelluft volumes were defined as the incremental volumes of the $\mathrm{V}_{\mathrm{T}}$ at the $\mathrm{D}$ lung region from the basal $V_{T}$ without inspiratory efforts in each ventilatory setting. However, because the ventilatory settings affected the levels of total $\mathrm{V}_{\mathrm{T}}$ themselves, it was difficult to match the basal ventilatory status with several ventilatory settings. Therefore, similar to a common clinical scenario, the changes in the $V_{T}$ at the $D$ lung region caused by the adjustments using each single inspiratory setting were examined. The severity of pendelluft was assessed by comparing $\mathrm{V}_{\mathrm{T}}$ at the $\mathrm{D}$ lung region using several ventilatory settings.

Varying inspiratory flow in volume control ventilation and varying inspiratory pressure in pressure control ventilation or pressure support ventilation would modify the pendelluft by changing all inspiratory effort levels. Unfortunately, determining the entire inspiratory level settings of model lungs was difficult during simulation.
Therefore, simple volume control ventilation or pressure support ventilation and pressure control ventilation settings were used in this study. Further animal or human examination is necessary to determine the effects of varying inspiratory flow in volume control ventilation and varying inspiratory pressure in pressure control ventilation or pressure support ventilation.

Because it is extremely difficult to assess ventilatory gas distribution status in the lungs using several ventilatory settings in humans or animals, we used lung models in this study. However, the use of such models, rather than human or animal models, is a major limitation of this study. The regional lung mechanics are an important factor affecting ventilatory gas distribution. Some researchers have measured regional lung compliances using electro-impedance tomography. ${ }^{3-5}$ Becher et $\mathrm{al}^{4}$ evaluated regional lung compliances during assisted spontaneous breathing in subjects admitted to the ICU. The mean \pm SD regional lung compliance of the ventral lung region was $40.9 \pm 15.9 \mathrm{~mL} / \mathrm{cm}$ $\mathrm{H}_{2} \mathrm{O}$, and that of the dorsal lung region was $18.8 \pm 9.3$ $\mathrm{mL} / \mathrm{cm} \mathrm{H}_{2} \mathrm{O}$. Moreover, Spadaro et $\mathrm{al}^{3}$ measured regional lung compliances in the $\mathrm{D}$ and ND lung regions in 14 


\section{Ventilatory Settings and Pendelluft Phenomenon}

subjects with acute hypoxic respiratory failure and ARDS. They reported that median (interquartile range) regional lung compliances in the $\mathrm{ND}$ and the $\mathrm{D}$ lung regions were $25.3(21.3-30.4)$ and $11.9(10.4-16.7) \mathrm{mL} / \mathrm{cm} \mathrm{H}_{2} \mathrm{O}$, respectively. The settings used in our study were similar to those in previous studies. ${ }^{3}$

The settings of the $\mathrm{P}_{\mathrm{pl}}$ in each lung region are another limitation of this study. The diaphragmatic bellows, which were ventilated with the Avea ventilators, produced 4 different $P_{p l}$ levels. The lowest and highest levels of $P_{p l}$ were used in the experiments to examine the effects of the ventilatory settings. The $\mathrm{P}_{\mathrm{pl}}$ at the $\mathrm{D}$ lung region was 2 times higher than that at the ND lung region in previous animal experiments. However, the driving pressure of the ventilator at the D lung region was 4-fold higher than that at the ND lung region in our study, and the level of driving pressure was not parallel to $\mathrm{P}_{\mathrm{pl}}{ }^{1}$ Therefore, the settings of the lung models were evaluated to establish pendelluft. To validate the results of our study, well-designed studies must be conducted in the future.

\section{Conclusions}

We established a lung model for pendelluft. Pendelluft was affected by the heterogeneity of lung mechanics and negative $\mathrm{P}_{\mathrm{pl}}$ between the ND and D lung regions. Minimal association was observed between the severity of pendelluft and the ventilator settings, such as ventilatory mode, triggering sensitivity, inspiratory pressurization, and inspiratory cycling-off. Moreover, no individual setting eliminated pendelluft.

\section{REFERENCES}

1. Yoshida T, Torsani V, Gomes S, De Santis RR, Beraldo MA, Costa ELV, et al. Spontaneous effort causes occult pendelluft during mechanical ventilation. Am J Respir Crit Care Med 2013;188(12):1420-1427.
2. Koyama Y, Uchiyama A, Yoshida J, Yoshida T, Yamashita T, Fujino $\mathrm{Y}$. A comparison of the adjustable ranges of inspiratory pressurization during pressure controlled continuous mandatory ventilation of 5 ICU ventilators. Respir Care 2018;63(7):849-858.

3. Spadaro S, Mauri T, Böhm SH, Scaramuzzo G, Turrini C, Waldmann $\mathrm{AD}$, et al. Variation of poorly ventilated lung units (silent spaces) measured by electrical impedance tomography to dynamically assess recruitment. Crit Care 2018;22(1):26-34.

4. Becher TH, Bui S, Zick G, Bläser D, Schädler D, Weiler N, Frerichs I. Assessment of respiratory system compliance with electrical impedance tomography using a positive end-expiratory pressure wave maneuver during pressure support ventilation: a pilot clinical study. Crit Care 2014;18(6):679-689.

5. Dargaville PA, Rimensberger PC, Frerichs I. Regional tidal ventilation and compliance during a stepwise vital capacity manoeuvre. Intensive Care Med 2010;36(11):1953-1961.

6. Yoshida T, Uchiyama A, Mashimo T, Fujino Y. The effect of ventilator performance on airway pressure release ventilation: a model lung study. Anesth Analg 2011;113(3):529-533.

7. Amato MBP, Marini JJ. Pressure-controlled and inverse-ratio ventilation. In: Tobin MJ. Principle and practice of mechanical ventilation, 3rd ed. New York: McGraw-Hill; 2012:227-252.

8. Uchiyama A, Imanaka H, Taenaka N, Nakano S, Fujino Y, Yoshiya I. A comparative evaluation of pressure-triggering and flow-triggering in pressure support ventilation (PSV) for neonates using an animal model. Anaesth Intensive Care 1995;23(3):302-306.

9. Chatburn RL. Classification of mechanical ventilators and modes of ventilation. In: Tobin MJ. Principle and practice of mechanical ventilation, 3rd ed. New York: McGraw-Hill; 2012: 45-64.

10. Uchiyama A, Imanaka H, Taenaka N. Relationship between work of breathing provided by a ventilator and patients' inspiratory drive during pressure support ventilation: effects of inspiratory rise time. Anaesth Intensive Care 2001;29(4):349-358.

11. Chiumello D, Pelosi P, Croci M, Bigatello LM, Gattinoni L. The effects of pressurization rate on breathing pattern, work of breathing, gas exchange, and patient comfort in pressure support ventilation. Eur Respir J 2001;18(1):107-114

12. Chiumello D, Pelosi P, Taccone P, Slutsky A, Gattinoni L. Effect of different inspiratory rise time and cycling off criteria during pressure support ventilation in patients recovering from acute lung injury. Crit Care Med 2003;31(11):2604-2610.

This article is approved for Continuing Respiratory Care Education credit. For information and to obtain your CRCE

(free to AARC members) visit

www.rcjournal.com 